Gefässchirurgie 2009 · 14:477-477

DOI 10.1007/s00772-009-0708-y

Online publiziert: 8. November 2009

(c) Springer Medizin Verlag 2009

\author{
H.-J. Hermanns \\ Praxis für Gefäßmedizin und Helios Klinik Hüls, Krefeld
}

\title{
Adipositas und Gefäßchirurgie
}

kungen. 5 Mrd. Euro sind allein für die Behandlung des Diabetes mellitus (Typ 2) aufzuwenden.

In Kombination mit arterieller Hypertonie, Diabetes mellitus, Hypercholesterinämie und Rauchen ist die Adipositas maßgeblich an der Entstehung von kardiovaskulären Erkrankungen beteiligt.

\section{( 15 Mio. Bundesbürger tragen 400 Mio. Kilogramm Übergewicht mit sich herum}

Bei einigen chronischen Erkrankungen mit operativen Maßnahmen (Kardiovaskularchirurgie) scheint jedoch ein moderates Übergewicht („obesity paradox“) die Überlebensrate sogar zu verbessern, insbesondere im Vergleich zu Untergewichtigen $[1,2,3]$.

Welche Rolle Adipositas und BMI in der Entwicklung von Gefäßkrankheiten spielen und wie sich das therapeutische Ergebnis nach vaskulären Eingriffen darstellt, wird Inhalt dieses Leitthemenkomplexes sein.
Auch die Entwicklung im Kindesalter ist bedenklich: 1,8 Mio. (13\%) deutsche Kinder werden bereits als übergewichtig eingestuft. Eine Ernährungsberatung für Kinder und mehr Bewegungstherapie im Schulunterricht sind dringend erforderlich.

Die gesundheitsökonomischen Folgen sind alarmierend. Schätzungsweise 40 Mrd. Euro Kosten pro Jahr entstehen aufgrund adipositasassoziierter Erkran-

\section{Korrespondenzadresse}

\section{Dr. H.-J. Hermanns}

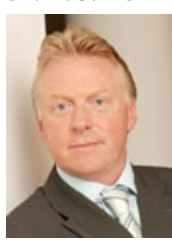

Praxis für Gefäßmedizin

und Helios Klinik Hüls

Neue Linner Str. 86

47799 Krefeld

hermanns@gefaessmedizin.info

\section{Literatur}

1. Curtis JP, Selter JG, Younfei W et al (2005) The obesity paradox. Body mass index and outcomes in patients with heart failure. Arch Int Med 265:5561

2. Lavi CJ, Osman AF, Milani RV, Mehra M (2003) Body composition and prognosis in chronic systolic heart failure: The obesity paradox. Am J Cardiol 91:891-894

3. Pingitore A, DiBella G, Lombardi M et al (2007) The obesity paradox and myocardial infarct size. J Cardiovasc Med 8:713-717

4. Statistisches Bundesamt Deutschland, Wiesbaden: http://www.destatis.de

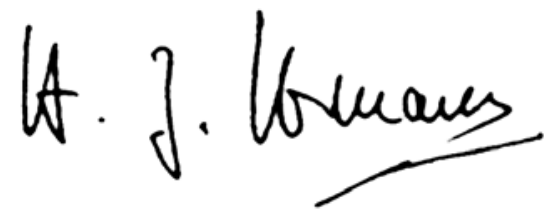

H.-J. Hermanns 